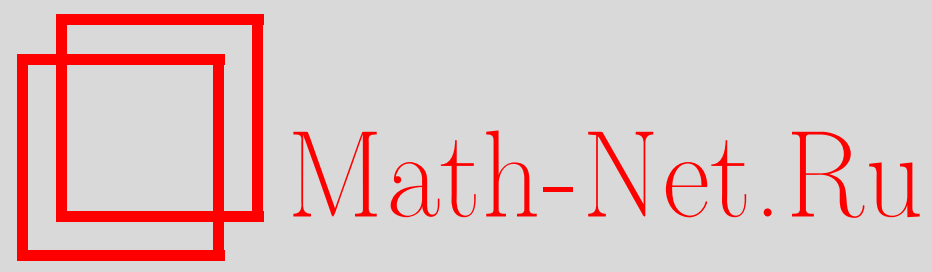

А. А. Пекарский, Новое доказательство неравенства Семмеса для производной рациональной функции, Матем. заметки, 2002, том 72, выпуск 2, 258-264

DOI: https://doi.org/10.4213/mzm419

Использование Общероссийского математического портала Math-Net.Ru подразумевает, что вы прочитали и согласны с пользовательским соглашением http://www . mathnet.ru/rus/agreement

Параметры загрузки:

IP : 35.173 .219 .149

26 апреля 2023 г., $16: 22: 28$

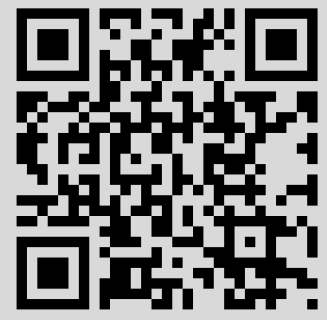




\section{НОВОЕ ДОКАЗАТЕЛЬСТВО НЕРАВЕНСТВА СЕММЕСА ДЛЯ ПРОИЗВОДНОЙ РАЦИОНАЛЬНОЙ ФУНКЦИИ}

\section{А. А. Пекарский}

В открытом круге $|z|<1$ комплексной плоскости рассматриваются следующие пространства функций: $\mathscr{B}$-пространство Блоха; $H_{p}^{\alpha}, \alpha \geqslant 0,0<p \leqslant \infty,-$ пространство Харди-Соболева; $B_{p}^{\alpha}, \alpha \geqslant 0,0<p \leqslant \infty,-$ пространство Харди-Бесова. Показано, что если все полюсы рациональной функции $R$ степени $n, n=1,2,3, \ldots$, лежат в области $|z|>1$, то $\|R\|_{H_{1 / \alpha}^{\alpha}} \leqslant c n^{\alpha}\|R\|_{\mathscr{B}},\|R\|_{B_{1 / \alpha}^{\alpha}} \leqslant c n^{\alpha}\|R\|_{\mathscr{B}}$, где $\alpha>0$, а $c>0$ зависит лишь от $\alpha$. Второе из этих неравенств в случае полуплоскости было получено Семмесом в 1984 году. Доказательство Семмеса основано на ганкелевых операторах, а наше - на специальном интегральном представлении рациональной функции.

Библиограффия: 7 названий.

Семмес [1], используя ганкелевы операторы, получил (см. ниже (2)) неравенство типа Бернштейна, связываюшее квазинормы рациональной функции (р.ф.) в пространствах Блоха $\mathscr{B}$ и Харди-Бесова $B_{1 / \alpha}^{\alpha}$. В связи с этим см. также работы Пеллера [2], [3], Рохберга [4] и автора [5]. Здесь предлагается прямое, существенно более простое доказательство неравенства (2). Наше доказательство основано на специальном интегральном представлении р.ф. (см. ниже лемма 1). Это позволяет, с одной стороны, существенно упростить доказательство результатов из [1] и [4] о наилучших рациональных приближениях в пространстве Блоха. С другой стороны, наше доказательство показывает, что указанные результаты можно получить без обращения к теории ганкелевых операторов. Отметим еще, что в [1] неравенство (2) доказано для полуплоскости, а мы проводим его доказательство для круга.

Введем необходимые обозначения. Пусть $D$ и $T$ суть соответственно открытьй круг $|z|<1$ и его граница - окружность $|z|=1$. Через $m_{1}$ и $m_{2}$ обозначим соответственно меру Лебега, рассматриваемую на жордановых спрямляемых кривых $S \subset \mathbb{C}$, и плоскую меру Лебега в $\mathbb{C}$. Через $L_{p}(S), 0<p \leqslant \infty$, обозначим пространство Лебега комплексных функций на $S$, наделенных стандартной квазинормой $\|\cdot\|_{p}=\|\cdot\|_{L_{p}(S)} \cdot$

Для функции $f$, аналитической в $D$, и $\alpha>0$ через $\mathscr{J}^{\alpha} f$ обозначаем производную Римана-Лиувилля порядка $\alpha$. Именно, если $\hat{f}(k), k=0,1,2, \ldots$, есть $k$-й коэффициент Маклорена $f$, то $\left(\mathscr{J}^{\alpha} f\right)^{\wedge}(k)=(\Gamma(k+\alpha+1) / \Gamma(k+1)) \hat{f}(k)$, где $\Gamma$ - гамма-функция Эйлера. Очевидно, $\left(\mathscr{J}^{n} f\right)(z)=\left(z^{n} f(z)\right)^{(n)}$ для $n \in \mathbb{N}$. Будем также считать $\mathscr{J}^{0} f:=f$ и $\mathscr{J} f:=\mathscr{J}^{1} f$.

В работе рассматриваются следующие пространства функций, аналитических в $D$ : $H_{p}, 0<p \leqslant \infty,-$ пространство Харди (см., например, [6]); $H_{p}^{\alpha}, \alpha \geqslant 0,0<p \leqslant \infty,-$ пространство Харди-Соболева; $B_{p}^{\alpha}, \alpha \geqslant 0,0<p \leqslant \infty,-$ пространство Харди-Бесова; 
$\mathscr{B}$ - пространство Блоха. Именно, $f \in H_{p}^{\alpha}$, если $\mathscr{J}^{\alpha} f \in H_{p}$. При этом полагаем $\|f\|_{H_{p}^{\alpha}}=\left\|\mathscr{J}^{\alpha} f\right\|_{H_{p}}$. Далее, $f \in B_{p}^{\alpha}$, если конечна квазинорма

$$
\begin{aligned}
\|f\|_{B_{p}^{\alpha}}:= & {\left[\frac{1}{\pi} \int_{D}\left|\left(\mathscr{J}^{\alpha+1} f\right)(z)\right|^{p}\left(1-|z|^{2}\right)^{p-1} d m_{2}(z)\right]^{1 / p} \text { при } 0<p<\infty ; } \\
& \|f\|_{B_{\infty}^{\alpha}}:=\sup _{z \in D}\left|\left(\mathscr{J}^{\alpha+1} f\right)(z)\right|\left(1-|z|^{2}\right) \quad \text { при } p=\infty .
\end{aligned}
$$

Пространство $B_{\infty}^{0}$ называется пространством Блоха и обозначается через $\mathscr{B}$.

Положительные величины, в разных местах, вообе говоря, разные, обозначаем через $c, c_{1}, c_{2}, \ldots ; c(\ldots), c_{1}(\ldots), c_{2}(\ldots), \ldots$.

Основным результатом данной статьи является следующая

Теорема 1. Пусть $R$ - рациональная функиия степени $n, n=1,2,3, \ldots$, все полюсы которой лежат вне круга $\bar{D}$. Тогда

$$
\begin{gathered}
\|R\|_{H_{1 / \alpha}^{\alpha}} \leqslant c_{1}(\alpha) n^{\alpha}\|R\|_{\mathscr{B}}, \\
\|R\|_{B_{1 / \alpha}^{\alpha}} \leqslant c_{2}(\alpha) n^{\alpha}\|R\|_{\mathscr{B}} .
\end{gathered}
$$

Как известно (см., например, [5]), $H_{2}^{\alpha}=B_{2}^{\alpha}, H_{p}^{\alpha} \varsubsetneqq B_{p}^{\alpha}$ при $p>2$ и $H_{p}^{\alpha} \supsetneqq B_{p}^{\alpha}$ при $p<2$. Поэтому неравенства (1) и (2) равносильны при $\alpha=1 / 2$; (1) сильнее (2) при $\alpha<1 / 2$ и (2) сильнее (1) при $\alpha>1 / 2$.

Оставшаяся часть работы посвяшена доказательству теоремы 1.

Нам понадобиться следующая формула типа формул Джрбашяна и Бергмана: если функиия $f$ аналитична в $D$ и $(\xi f(\xi))^{\prime} \ln (1 /|\xi|)$ суммируема в $D$, то

$$
f(z)=\frac{2}{\pi} \int_{D} \frac{(\xi f(\xi))^{\prime} \ln (1 /|\xi|)}{(1-\bar{\xi} z)^{2}} d m_{2}(\xi), \quad z \in D .
$$

В справедливости (3) можно убедиться по следующей схеме:

1) используя полярные координаты, доказать (3) для функции $f(z)=z^{n}, n=0,1$, $2, \ldots ;$

2) доказать (3) для полиномов;

3) с помошью предельного перехода получить (3) в общем виде.

Для $a \in D$ введем аналитическую в $D$ функцию $\psi_{a}(z)=(1-\bar{a} z)^{-1}$. Сравнивая коэффициенты при одинаковых степенях $z$, находим, что при $\alpha \geqslant 0$

$$
\left(\mathscr{J}^{\alpha} \psi_{a}\right)(z)=\Gamma(\alpha+1)(1-\bar{a} z)^{-\alpha-1},
$$

где ветвь многозначной функции $(1-\xi)^{-\alpha-1}$ выбрана так, что $(1-\xi)^{-\alpha-1}>0$ при $\xi \in(-\infty, 1)$.

Пусть $R$ - р.ф. из теоремы 1 . Ее полюсы запишем в виде $1 / \bar{a}_{k}, k=1, \ldots, n$, где $a_{k} \in D$. Введем

$$
B(z):=\prod_{k=0}^{n} \frac{z-a_{k}}{1-\bar{a}_{k} z}, \quad a_{0}:=0,
$$

- произведение Бляшке порядка $n+1$. 
Лемма 1. Для любых $\alpha>0 u z \in \bar{D}$ имеет место равенство

$$
\left(\mathscr{J}^{\alpha} R\right)(z)=\frac{2 \Gamma(\alpha+1)}{\pi} \int_{D}(\xi R(\xi))^{\prime}\left[\frac{\partial}{\partial \bar{\xi}} \bar{\xi}\left(\frac{1-\overline{B(\xi)} B(z)}{1-\bar{\xi} z}\right)^{\alpha+1}\right] \ln \frac{1}{|\xi|} d m_{2}(\xi) .
$$

ДокАЗАТЕЛЬСТво. При фиксированном $z \in \bar{D}$ обе части (5) непрерьвно зависят от полюсов $R$. Следовательно, можем считать числа $a_{0}, a_{1}, a_{2}, \ldots, a_{n}$ попарно различными. Тогда, раскладывая $R$ на простые дроби, будем иметь

$$
R(z)=\sum_{k=0}^{n} b_{k} \psi_{a_{k}}(z)
$$

где $b_{k}$ - некоторые коэффициенты. Поэтому нам достаточно убедиться в справедливости (5) для каждой функции $R=\psi_{a_{k}}$. Пусть $g_{a_{k}}-$ правая часть $(5)$ с $\psi_{a_{k}}$ вместо $R$. Поскольку $\left(\xi \psi_{a_{k}}(\xi)\right)^{\prime}=\left(1-\bar{a}_{k} \xi\right)^{-2}$, то

$$
\overline{g_{a_{k}}(z)}=\frac{2 \Gamma(\alpha+1)}{\pi} \int_{D}\left[\frac{\partial}{\partial \xi} \xi\left(\frac{1-B(\xi) \overline{B(z)}}{1-\xi \bar{z}}\right)^{\alpha+1}\right] \frac{\ln (1 /|\xi|)}{\left(1-\bar{\xi} a_{k}\right)^{2}} d m_{2}(\xi)
$$

С учетом (3) и равенства $B\left(a_{k}\right)=0$ отсюда получим, что $\overline{g_{a_{k}}(z)}=\Gamma(\alpha+1)\left(1-\bar{z} a_{k}\right)^{-\alpha-1}$, т.е. $($ см. $(4)) g_{a_{k}}(z)=\left(\mathscr{J}^{\alpha} \psi_{a_{k}}\right)(z)$. Лемма 1 доказана.

Из леммы 1 и неравенства $\rho \ln (1 / \rho) \leqslant\left(1-\rho^{2}\right) / 2,0<\rho \leqslant 1$, получаем, что для любых $a>0$ и $z \in \bar{D}$

$$
\left|\left(\mathscr{J}^{\alpha} R\right)(z)\right| \leqslant \frac{\Gamma(\alpha+1)\|R\|_{\mathscr{B}}}{\pi} \int_{D}\left|\frac{\partial\left(\xi Q(\xi, z)^{\alpha+1}\right)}{\partial \xi}\right| \frac{d m_{2}(\xi)}{|\xi|},
$$

где

$$
Q(\xi, z):=\frac{1-\overline{B(z)} B(\xi)}{1-\bar{z} \xi} .
$$

Несложно убедиться, что при всех $\xi$ и $z$ из $\bar{D}$ выполняется соотношение

$$
\left|\frac{\partial\left(\xi Q(\xi, z)^{\alpha+1}\right)}{\partial \xi}\right| \leqslant \frac{(\alpha+1)|Q(\xi, z)|^{\alpha}}{|1-\xi \bar{z}|}\left(\left|B^{\prime}(\xi)\right|+|Q(\xi, z)|\right) .
$$

Пусть $a \in D$ и $\alpha>0$. В дальнейшем неоднократно будут применяться неравенства

$$
\begin{gathered}
\int_{\Gamma} \frac{d m_{1}(\eta)}{|1-\bar{a} \eta|^{\alpha+1}} \leqslant \frac{c_{1}(\alpha)}{(1-|a|)^{\alpha}}, \\
\int_{D} \frac{d m_{2}(\xi)}{|1-\bar{a} \xi|^{\alpha+2}|\xi|} \leqslant \frac{c_{2}(\alpha)}{(1-|a|)^{\alpha}} .
\end{gathered}
$$

Соотношение (8) доказано в [5] и [7, гл. 10]. Для получения (9) нужно перейти к полярным координатам и воспользоваться (8).

Нам понадобится также радиальная максимальная функиия $M^{*}$. Именно, для функции $f$, определенной в $D$, положим

$$
\left(M^{*} f\right)(\eta):=\sup _{\rho \in(0,1)}|f(\rho \eta)|, \quad \eta \in T .
$$

Согласно теореме Харди-Литтлвуда (см., например, [6]) если $f \in H_{p}$ и $0<p<\infty$, то

$$
\int_{T}\left(M^{*} f\right)^{p} d m_{1} \leqslant c \int_{T}|f|^{p} d m_{1}
$$

Здесь под $f(\eta)$ для $f \in H_{p}$ и $\eta \in T$ подразумеваются некасательные предельные значения $f(z), z \in D$. 
ЛЕмма 2. Для любых $\alpha>0 u z \in T$ выполняется неравенство

$$
\left|\left(\mathscr{J}^{\alpha} R\right)(z)\right| \leqslant c(\alpha)\|R\|_{\mathscr{B}} \int_{T}\left|\frac{\partial\left(\xi Q(\xi, z)^{\alpha+1}\right)}{\partial \xi}\right|^{(\alpha+1) /(\alpha+2)} d m_{1}(\xi)
$$

ДокАЗАТЕЛЬСТво. Для фиксированного $z \in T$ введем функцию

$$
f_{z}(\xi)=\frac{\partial\left(\xi Q(\xi, z)^{\alpha+1}\right)}{\partial \xi}
$$

принадлежащую всем классам $H_{p}$. Из $(7)$ следует, что $\left|f_{z}(\xi)\right| \leqslant 6^{\alpha} /(1-|\xi|)^{\alpha+2}$. Поэтому, переходя к полярным координатам $\xi=\rho \eta, 0 \leqslant \rho<1, \eta \in T$, из (6) получим

$$
\left|\left(\mathscr{J}^{\alpha} R\right)(z)\right| \leqslant c_{1}(\alpha)\|R\|_{\mathscr{B}} \int_{T} d m_{1}(\eta) \int_{0}^{1} \min \left\{\frac{1}{(1-\rho)^{\alpha+2}},\left(M^{*} f_{z}\right)(\eta)\right\} d \rho .
$$

Следовательно,

$$
\left|\left(\mathscr{J}^{\alpha} R\right)(z)\right| \leqslant c_{2}(\alpha)\|R\|_{\mathscr{B}} \int_{T}\left(M^{*} f_{z}\right)(\eta)^{(\alpha+1) /(\alpha+2)} d m_{1}(\eta) .
$$

Остается воспользоваться неравенством (10). Лемма 2 доказана.

В связи с леммой 2 и неравенством (7) на окружности $T$ рассмотрим следуюшие функции (определенные при указанных значениях параметров $\beta, \gamma$ и $\delta$ ):

$$
\begin{aligned}
K(z, \beta) & :=\int_{T}|Q(\eta, z)|^{\beta} d m_{1}(\eta), \quad \beta>1 ; \\
N(z, \beta, \gamma) & :=\int_{T} \frac{|Q(\eta, z)|^{\beta}}{|1-\bar{z} \eta|^{\gamma}} d m_{1}(\eta), \quad \beta>0, \quad 0<\gamma<1, \quad \beta+\gamma>1 ; \\
S(z, \beta, \gamma) & :=\int_{T} \frac{|Q(\eta, z)|^{\beta}}{|1-\bar{z} \eta|^{\gamma}}\left|B^{\prime}(\eta)\right|^{\gamma} d m_{1}(\eta), \quad \beta>0, \quad 0<\gamma<1, \quad \beta+\gamma>1 ; \\
\Lambda(z, \delta) & :=\sum_{k=0}^{n} \frac{1}{\left|1-\bar{a}_{k} z\right|}\left(\frac{1-\left|a_{k}\right|}{\left|1-\bar{a}_{k} z\right|}\right)^{\delta}, \quad \delta>0 .
\end{aligned}
$$

Для функции $K$ справедлива оценка (см. [5] или [7, гл. 10]):

$$
K(z, \beta) \leqslant c(\beta) \Lambda\left(z, \frac{1}{\beta+1}\right)^{\beta-1}, \quad z \in T .
$$

В следующих леммах 3 и 4 получены необходимые нам соотношения для функций $N$ и $S$.

ЛЕмма 3. Для любых $z \in T$ выполняется неравенство

$$
N(z, \beta, \gamma) \leqslant c(\beta, \gamma) \Lambda(z, \delta)^{\beta+\gamma-1}
$$

əде $\delta=\delta(\beta, \gamma) \in(0,1)$. 
ДоКАЗАТЕЛЬСТВО. Поскольку $\beta>0$ и $0<\gamma<1$, то можем подобрать положительные числа $p$ и $q$, удовлетворяющие условиям $1 / p+1 / q=1, p \gamma<1$ и $q \beta>1$. Положим $\delta=1 /(q \beta+1)$ и $E(z)=\{\eta \in T:|1-\bar{z} \eta| \leqslant 1 / \Lambda(z, \delta)\}$. Используя неравенство Гёльдера, находим, что вклад в интеграл от $N$ по множеству $E(z)$ не превосходит

$$
\begin{aligned}
& \left(\int_{E(z)} \frac{d m_{1}(\eta)}{|1-\bar{z} \eta|^{p \gamma}}\right)^{1 / p}\left(\int_{E(z)}|Q(\eta, z)|^{q \beta} d m_{1}(\eta)\right)^{1 / p} \leqslant c_{1} \Lambda(z, \delta)^{\gamma-1 / p} K(z, q \beta)^{1 / q} \\
& \quad \leqslant c_{2} \Lambda(z, \delta)^{\beta+\gamma-(1 / p+1 / q)}=c_{2} \Lambda(z, \delta)^{\beta+\gamma-1} .
\end{aligned}
$$

Здесь при получении последнего неравенства мы воспользовались соотношением (11).

Для оценки вклада в интеграл от $N$ по множеству $T \backslash E(z)$ воспользуемся неравенством $|Q(\xi, z)| \leqslant 2 /|1-\bar{z} \xi|, z, \xi \in T$. С учетом условия $\beta+\gamma>1$ находим, что указанньй вклад не превосходит

$$
\int_{T \backslash E(z)} \frac{2^{\beta}}{|1-\bar{z} \eta|^{\beta+\gamma}} d m_{1}(\eta) \leqslant c_{3} \Lambda(z, \delta)^{\beta+\gamma-1} .
$$

Этим лемма 3 доказана.

ЛЕмМА 4. Имеет место неравенство

$$
\int_{T} S(z, \beta, \gamma)^{1 /(\beta+2 \gamma-1)} d m_{1}(z) \leqslant c(\beta, \gamma) n .
$$

ДокАЗАТЕЛЬСТво. Для $\eta \in T$ и $\delta \in(0,1]$ имеем

$$
\left|B^{\prime}(\eta)\right|=\sum_{k=0}^{n} \frac{1-\left|a_{k}\right|^{2}}{\left|1-\bar{a}_{k} \eta\right|^{2}} \leqslant 2 \Lambda(\eta, 1) \leqslant 2 \Lambda(\eta, \delta) .
$$

Поэтому, используя теорему Фубини, лемму 3 , неравенства (8) и $(11)$, получим искомое утверждение в случае $\beta+2 \gamma=2$ :

$$
\begin{aligned}
\int_{T} S(z, \beta, \gamma) d m_{1}(z) & =\int_{T} N(\eta, \beta, \gamma)\left|B^{\prime}(\eta)\right|^{\gamma} d m_{1}(\eta) \\
& \leqslant c_{1} \int_{T} \Lambda(\eta, \delta)^{\beta+\gamma-1} \Lambda(\eta, \delta)^{\gamma} d m_{1}(\eta) \leqslant c_{2} n .
\end{aligned}
$$

Общий случай сведем к рассмотренному частному случаю. С этой целью выберем положительные числа $\beta_{0}, \beta_{1}, p_{0}$ и $p_{1}$, удовлетворяющие условиям $\beta_{0}+\beta_{1}=\beta, 1 / p_{0}+1 / p_{1}=1$, $p_{0} \beta_{0}>1$ и $p_{1}\left(\beta_{1}+2 \gamma\right)=2$. Существование таких чисел следует из наложенных на $\beta$ и $\gamma$ ограничений. Из неравенства Гёльдера получаем

$$
S(z, \beta, \gamma) \leqslant K\left(z, \beta_{0}, p_{0}\right)^{1 / p_{0}} S\left(z, p_{1} \beta_{1}, p_{1} \gamma\right)^{1 / p_{1}} .
$$

Далее заметим, что

$$
\beta+2 \gamma-1=\left(\beta_{0}-\frac{1}{p_{0}}\right)+\frac{1}{p_{1}} .
$$

Поэтому можем применить неравенство Гёльдера в следующей форме:

$$
\|S(\cdot, \beta, \gamma)\|_{1 /(\beta+2 \gamma-1)} \leqslant\left\|K\left(\cdot, p_{0} \beta_{0}\right)\right\|_{1 /\left(p_{0} \beta_{0}-1\right)}^{1 / p_{0}}\left\|S\left(\cdot, p_{1} \beta_{1}, p_{1} \gamma\right)\right\|_{1}^{1 / p_{1}} .
$$

Согласно $(8),(11)$ и отмеченному вьше частному случаю (в данной ситуации $p_{1} \beta_{1}+$ $2 p_{1} \gamma=2$ ) получим

$$
\|S(\cdot, \beta, \gamma)\|_{1 /(\beta+2 \gamma-1)} \leqslant c_{3} n^{\beta_{0}-1 / p_{0}} n^{1 / p_{1}} .
$$

Остается еще раз воспользоваться равенством (12). Лемма 4 доказана. 
ДОКАЗАТЕЛЬСТВО НЕРАВЕНСТВА (1). Используя лемму 2 и неравенство (7), получаем, что при $z \in T$

$$
\left|\left(\mathscr{J}^{\alpha} R\right)(z)\right| \leqslant c(\alpha)\|R\|_{\mathscr{B}}\left\{N\left(z, \frac{(\alpha+1)^{2}}{\alpha+2}, \frac{\alpha+1}{\alpha+2}\right)+S\left(z, \frac{\alpha(\alpha+1)}{\alpha+2}, \frac{\alpha+1}{\alpha+2}\right)\right\} .
$$

Здесь функция $N$ удовлетворяет указанньм вьше ограничениям на параметры при всех $\alpha>0$, а функция $S$ - лишь при $\alpha>(\sqrt{5}-1) / 2$. Поэтому из лемм 3 и 4 мы получим неравенство $(1)$ для $\alpha>(\sqrt{5}-1) / 2$.

Пусть сейчас $0 \leqslant \alpha \leqslant(\sqrt{5}-1) / 2=0.61 \ldots$ Заметим, что при $\alpha \in(0,1)$ имеет место равенство

$$
\left(\mathscr{J}^{\alpha} R\right)(\eta)=\frac{1}{\Gamma(1-\alpha)} \int_{0}^{1}(\mathscr{J} R)(\rho \eta) \rho^{\alpha}(1-\rho)^{-\alpha} d \rho, \quad \eta \in T .
$$

Для его доказательства нужно разложить $R$ в ряд Маклорена и почленно проинтегрировать.

Из определений нормы в пространстве $\mathscr{B}$ и радиальной максимальной функции $M^{*}$ для $\rho \in[0,1)$ и $\eta \in T$ получаем, что

$$
|(\mathscr{J} R)(\rho \eta)| \leqslant \min \left\{\frac{\|R\|_{\mathscr{B}}}{1-\rho},\left(M^{*} \mathscr{J} R\right)(\eta)\right\} .
$$

С учетом (13) отсюда находим

$$
\left|\left(\mathscr{J}^{\alpha} R\right)(\eta)\right| \leqslant \frac{1}{\Gamma(1-\alpha)} \int_{0}^{1} \min \left\{\frac{\|R\|_{\mathscr{B}}}{1-\rho},\left(M^{*} \mathscr{J} R\right)(\eta)\right\} \frac{d \rho}{(1-\rho)^{\alpha}} .
$$

Следовательно,

$$
\left|\left(\mathscr{J}^{\alpha} R\right)(\eta)\right| \leqslant c(\alpha)\|R\|_{\mathscr{B}}^{1-\alpha}\left(M^{*} \mathscr{J} R\right)(\eta)^{\alpha} .
$$

Остается воспользоваться соотношением (10) и тем, что для $\alpha=1$ неравенство (1) уже получено. Неравенство (1) доказано полностью.

ДокАЗАТЕЛЬСТво НЕРАВенСтВА (2). Для $\eta \in T$ и $\rho \in[0,1)$ имеют место соотношения

$$
\begin{aligned}
& \left|\left(\mathscr{J}^{\alpha+1} R\right)(\rho \eta)\right| \leqslant\left(M^{*} \mathscr{J}^{\alpha+1} R\right)(\eta), \\
& \left|\left(\mathscr{J}^{\alpha+1} R\right)(\rho \eta)\right| \leqslant \frac{c_{1}(\alpha)\|R\|_{\mathscr{B}}}{(1-\rho)^{\alpha+1}} .
\end{aligned}
$$

Первое из них следует из определения функции $M^{*}$. Для доказательства второго заметим, что $\left(\mathscr{J}^{\alpha} \psi_{a}^{2}\right)(z)=\Gamma(\alpha+1)(1+\alpha \bar{a} z)(1-\bar{a} z)^{-\alpha-2}$ (здесь $\psi_{a}$ функция из $\left.(4)\right)$. Значит, из (3) получим

$$
\left(\mathscr{J}^{\alpha} f\right)(z)=\frac{2 \Gamma(\alpha+1)}{\pi} \int_{D} \frac{1+\alpha \bar{\xi} z}{(1-\bar{\xi} z)^{\alpha+2}}(\xi f(\xi))^{\prime} \ln \frac{1}{|\xi|} d m_{2}(\xi), \quad z \in D,
$$

где функция $f$ удовлетворяет тем же условиям, что и в (3). Из (9) и (16) следует неравенство (15). 
Из определения (квази)нормы в $B_{1 / \alpha}^{\alpha},(14)$ и (15) следует соотношение

$$
\|R\|_{B_{1 / \alpha}^{\alpha}}^{1 / \alpha} \leqslant c_{2} \int_{T} d m_{1}(\eta) \int_{0}^{1} \min \left\{\frac{\|R\|_{\mathscr{B}}^{1 / \alpha}}{(1-\rho)^{2}}, \frac{\left(M^{*} \mathscr{J}^{\alpha+1} R\right)(\eta)^{1 / \alpha}}{(1-\rho)^{1-1 / \alpha}}\right\} d \rho .
$$

Вычисляя внутренний интеграл, получим, что

$$
\|R\|_{B_{1 / \alpha}^{\alpha}} \leqslant c_{3}\|R\|_{\mathscr{B}}^{1 /(\alpha+1)}\left[\int_{T}\left(M^{*} \mathscr{J}^{\alpha+1} R\right)(\eta)^{1 /(\alpha+1)} d m_{1}(\eta)\right]^{\alpha} .
$$

Остается воспользоваться соотношениями (10) и (1) (с $\alpha+1$ вместо $\alpha)$. Неравенство (2) доказано.

\section{СПИСОК ЦИТИРОВАННОЙ ЛИТЕРАТУРЫ}

[1] Semmes S. Trace ideal criteria for Hankel operators and application to Besov space // Integral Equations and Operator Theory. 1984. V. 7. P. 241-281.

[2] Пеллер В. В. Операторы Ганкеля класса $\sigma_{p}$ и их приложения (рациональная аппроксимация, гауссовские процессы, проблема мажорации операторов) // Матем. сб. 1980. Т. 113. № 4. C. $538-582$.

[3] Пеллер В. В. Описание операторов Ганкеля класса $\sigma_{p}$ при $p>0$, исследование скорости рациональной аппроксимации и другие приложения // Матем. сб. 1983. Т. 122. № 4. С. 481-510.

[4] Rochberg R. Decomposition theorems for Bergman spaces and their applications // Operator and Functional Theory. Proc. NATO Adv. Study Inst. (Lancaster, July 16-24, 1984): D. Reidel Publ. Company, 1985. P. 225-277.

[5] Пекарский А. А. Неравенства типа Бернштейна для производных рациональных функций и обратные теоремы рациональной аппроксимации // Матем. сб. 1984. Т. 124. № 4. С. 571-588.

[6] Гарнетт Дж. Ограниченные аналитические функции. М.: Мир, 1984.

[7] Lorentz G. G., Golitschek M. V., Makovoz Y. Constructive Approximation. Advanced Problems. Berlin: Spinger-Verlag, 1996.

Гродненский государственный университет им. Я. Купалы

Поступило

E-mail: vuv@univer.belpak.grodno.by

10.09.1998 